\title{
Effect of Barium Titanate Reinforcement on Tensile Strength and Dielectric Response of Electrospun Polyvinylidene Fluoride Fibers
}

\author{
Avinash Baji and Yiu-Wing Mai \\ Additional information is available at the end of the chapter \\ http://dx.doi.org/10.5772/intechopen.74662
}

\begin{abstract}
In this study, we used electrospinning to obtain polyvinylidene fluoride (PVDF) fibers reinforced with barium titanate $\left(\mathrm{BaTiO}_{3}\right)$ and investigated the influence of $\mathrm{BaTiO}_{3}$ concentration on the tensile strength and dielectric behavior of PVDF fibers. X-ray diffraction (XRD) study and infrared spectroscopy revealed that PVDF fibers filled with $\mathrm{BaTiO}_{3}$ possessed higher fraction of ferroelectric $\beta$-crystals compared to neat PVDF fibers. Further, incorporation of $40 \mathrm{wt} \% \mathrm{BaTiO}_{3}$ within the fibers increased their stiffness and strength by 95 and $38 \%$, respectively. These improvements in tensile properties of $\mathrm{BaTiO}_{3}$ filled PVDF fibers arose from the reinforcement effect of $\mathrm{BaTiO}_{3}$. Also, the dielectric response of the $\mathrm{BaTiO}_{3} / \mathrm{PVDF}$ fibers was characterized. The effective dielectric constants of PVDF fibers reinforced with $\mathrm{BaTiO}_{3}$ were found to increase consistently with $\mathrm{BaTiO}_{3}$ content at all frequencies. The dielectric loss of the fibers did not show any significant change for all concentrations of $\mathrm{BaTiO}_{3}$ within the fibers.
\end{abstract}

Keywords: electrospinning, nanofibers, polyvinylidene difluoride (PVDF), barium titanate $\left(\mathrm{BaTiO}_{3}\right)$, dielectric properties

\section{Introduction}

There has been substantial recent interest in the development of nanostructured piezo-sensitive composites as they can potentially display combination of desirable physical properties which cannot be obtained in single phase materials [1-3]. Hence, ceramics such as zinc oxide, bismuth ferrite, lead zirconate titanate, barium titanate $\left(\mathrm{BaTiO}_{3}\right)$ etc. are widely utilized in actuators, sensors and energy storage devices due to their good piezoelectric and ferroelectric 
properties [4-6]. However, their inherit brittleness has limited their use for most engineering applications. By contrast, compliant polymeric materials such as polyvinylidene fluoride (PVDF) and its copolymers are easy to process but generally have low piezoelectric coefficient values when compared to ferroelectric ceramics $[7,8]$. Thus, the incorporation of a ceramic phase into an electroactive polymeric matrix could yield composites with improved mechanical integrity and piezoelectric characteristics [1, 2].

Traditionally, such composites are fabricated by dispersing micron or submicron sized ferroelectric ceramics into a dielectric polymer matrix. However, this approach yields composite with film thickness greater than the size of the ceramic particles. Consequently, the composites are invariably found to possess low capacitance densities. Some recent studies demonstrate that reducing the size of the filler particles to nanometer length scales leads to an improvement in the dielectric permittivity of the composite [3,9]. This is attributed to an increase in the interfacial area which promotes the exchange couple effect, and improves the polarization levels of the composite and its dielectric response [3, 9]. Further, these studies demonstrate that electromechanical coupling and permittivity of the composite can increase by 60 times when large aspect ratio fillers are used and when they are aligned along the poling direction within the matrix phase $[10,11]$. Hence, composites reinforced with nanorods or nanowhiskers can display better dielectric permittivity and mechanical strength compared to composites obtained by simply dispersing ceramic powders within a polymer matrix [12-15].

It is well-known that these piezo-sensitive composites are often subjected to external stimuli such as mechanical stresses, electrical field or coupled electromechanical loads [16]. The induced electric field activates the mechanical stresses and the induced mechanical load generates the electrical field within the piezoelectric material [16]. Since the electric field and mechanical stresses interfere with each other, it is therefore of the utmost importance that the composites have good combined mechanical and piezoelectric responses. In our recent study, we fabricated $\mathrm{BaTiO}_{3}$ reinforced PVDF fibers using electrospinning and characterized their piezoresponse [2]. We showed that these $\mathrm{PVDF} / \mathrm{BaTiO}_{3}$ fibers have tremendous potential for future nanoscale electronic devices. Similarly, other researchers working on $\mathrm{PVDF} / \mathrm{BaTiO}_{3}$ composites focused their efforts to characterize the dielectric, piezoelectric and ferroelectric behaviors of the composites [7, 14, 15, 17].

In this work, the tensile deformation behavior of $\mathrm{PVDF} / \mathrm{BaTiO}_{3}$ fibers obtained using electrospinning is characterized. Their deformation mechanisms are discussed based on their microstructural evolution, such as crystalline structure development induced by the presence of $\mathrm{BaTiO}_{3}$ within the fibers. Finally, results on dielectric permittivity of these nanofibers are presented.

\section{Experimental work}

\section{1. $\mathrm{BaTiO}_{3}$ fibers}

We used sol-gel based electrospinning to prepare barium titanate fibers. Briefly, $5.1 \mathrm{~g}$ of barium acetate was dissolved in $12 \mathrm{ml}$ of glacial acetic acid; and to this solution, $5.9 \mathrm{ml}$ of titanium 
isopropoxide was added to obtain $\mathrm{BaTiO}_{3}$ precursor solution [18]. The solution for electrospinning was prepared by dissolving $2.5 \mathrm{~g}$ poly(vinyl pyrrolidone) ( $\mathrm{PVP}, \mathrm{MW}=360,000$ ) in $11 \mathrm{ml}$ of solvent solution which was prepared by mixing equal parts of dimethyl formamide (DMF) and ethanol together. Then, $\mathrm{BaTiO}_{3}$ precursor solution was added to the PVP solution.

Electrospinning was conducted using the $\mathrm{BaTiO}_{3} / \mathrm{PVP}$ solution at $20 \mathrm{kV}$ with $0.07 \mathrm{~mm} / \mathrm{min}$ as the solution feed rate. The spacing between needle and grounded metal collector was $15 \mathrm{~cm}$. A vacuum oven at $100^{\circ} \mathrm{C}$ was used for $1 \mathrm{~h}$ to dry the fibers. After which the fibers were put in a furnace and annealed at $750^{\circ} \mathrm{C}$ for $1 \mathrm{~h}$. The resultant fibers, referred to as Sample 1, were $\mathrm{BaTiO}_{3}$ fibers.

\section{2. $\mathrm{BaTiO}_{3}$ reinforced PVDF fibers}

PVDF fibers filled with $0,10,20$ and $40 \mathrm{wt} \%$ of $\mathrm{BaTiO}_{3}$ were obtained as described in the following steps. In the first step, Sample 1 fibers of known content were dispersed into DMF solution. The solution was sonicated for $0.5 \mathrm{~h}$ and then stirred for $1 \mathrm{~h}$ to obtain a slurry solution. $18 \mathrm{wt} \%$ of PVDF powder was added to the slurry solution for electrospinning.

Electrospinning was conducted at $18 \mathrm{kV}$ on this solution with a feed rate fixed at $0.12 \mathrm{~mm} /$ min. PVDF fibers filled with 0, 10, 20 and $40 \mathrm{wt} \%$ of $\mathrm{BaTiO}_{3}$ were obtained. This set of $\mathrm{BaTiO}_{3}-$ PVDF fiber samples are referred to as Sample 2.

\subsection{Microstructure characterization}

A scanning electron microscope (FESEM, Zeiss ULTRA plus) was used to observe the microstructure of Sample 1 and Sample 2 fibers. The surface of the samples were gold-coated with a sputter coater before they were examined using SEM. An accelerating voltage of $2-3 \mathrm{kV}$ was used for imaging the samples. Transmission electron microscopy (TEM, Philips CM120 Biofilter) was also used to image Sample 1 and Sample 2 fibers. Sample 2 fibers were electrospun directly on a 400-mesh copper grid and then examined using the TEM.

\subsection{X-ray analysis and infrared spectroscopy}

The diffraction behavior of Sample 1 and Sample 2 fibers were studied using an X-ray diffractometer (XRD Shimadzu S6000) with $\mathrm{Cu} \mathrm{K} \alpha$ radiation $(\lambda=1.54 \AA$ ). The $2 \theta$ scan was varied between 15 and $70^{\circ}$ and the scan speed was set at $1 \%$ min with $0.02^{\circ}$ step size. Bruker Fourier transform infrared spectroscopy system (FTIR, IFS 66v) was used to collect the spectra of Sample 2 fibers. The fibers were scanned from 5000 to $400 \mathrm{~cm}^{-1}$ in attenuated total reflectance mode (ATR).

\subsection{Mechanical properties}

The mechanical integrity of the fibers was analyzed using tensile tests on the aligned fiber samples conducted on an Instron 5567 ( $2.5 \mathrm{~N}$ load cell) testing machine with a cross-head speed of $5 \mathrm{~mm} / \mathrm{min}$. The loading direction was parallel to the fiber axis. 
Dynamic mechanical measurements on Sample 2 fibers were obtained by a dynamic mechanical analyzer (DMA, TA Instruments). An oscillation amplitude of $10 \mu \mathrm{m}, 3^{\circ} \mathrm{C} / \mathrm{min}$ heating ramp rate and $1 \mathrm{~Hz}$ frequency were used.

\subsection{Dielectric properties}

The frequency-dependent capacitance and loss tangent of Sample 2 fibers as a function of $\mathrm{BaTiO}_{3}$ content were measured using a frequency-response dielectric analyzer (Novocontrol alpha analyzer) with scanning frequencies ranging from 103 to $107 \mathrm{~Hz}$.

\section{Results and discussion}

Uniform distribution of the ferroelectric ceramic phase within the piezoelectric PVDF matrix is an important prerequisite to obtain electroactive composites with improved physical and mechanical properties, e.g., ferroelectricity, tensile strength, and stiffness [1, 11, 14]. Here, we use electrospinning to obtain PVDF fibers with uniformly dispersed and distributed $\mathrm{BaTiO}_{3}$. The effects of $\mathrm{BaTiO}_{3}$ loading on the structure development and tensile strength of the fibers are evaluated.

The microstructure and size of Samples 1 and 2 are examined using SEM and TEM. Figure 1A and $\mathbf{B}$ show typical SEM and TEM images of Sample $1 \mathrm{BaTiO}_{3}$ fiber indicating an average fiber diameter of $\sim 170 \pm 50 \mathrm{~nm}$. SEM image (Figure 1A) reveals that the fibers are composed of finegrained structures which are assembled and organized to obtain a fibrous geometry. These grains are dense and closely packed as evident in the TEM image (Figure 1B). Figure 2A and $\mathbf{B}$ show representative SEM and TEM microstructures of PVDF fibers filled with $20 \mathrm{wt} \%$ $\mathrm{BaTiO}_{3}$. An average fiber diameter of Sample 2 (Figure 2A) is $210 \pm 40 \mathrm{~nm}$. SEM image shows that the surface morphology of $\mathrm{BaTiO}_{3} / \mathrm{PVDF}$ fibers appears rough.

Arrows in Figure 2A clearly point to slight bulges in the fiber which are owing to the inclusions of $\mathrm{BaTiO}_{3}$ within the PVDF fibers. The inset in Figure 2A shows an array of uniaxial aligned and tightly packed fibers which can be easily collected to form a test coupon for tensile experiments. The dispersion quality of $\mathrm{BaTiO}_{3}$ in $\mathrm{PVDF}$ can be conveniently examined from the TEM image (Figure 2B). Consistent with the SEM images, bulges in the fiber are also noticed (see Figure 2B). A magnified TEM image taken from another area of the fiber is given in the inset of Figure 2B, which confirms the alignment of $\mathrm{BaTiO}_{3}$ along the fiber axis.

The crystalline phase change in PVDF due to the presence of $\mathrm{BaTiO}_{3}$ is determined using XRD and FTIR. Figure 3A shows the XRD patterns of PVDF fibers filled with 0, 10, 20 and $40 \mathrm{wt} \% \mathrm{BaTiO}_{3}$. For reference, the XRD pattern of neat $\mathrm{BaTiO}_{3}$ (Sample 1), that has a cubictetragonal structure which is responsible for its ferroelectric and dielectric properties [19, 20], is shown in Figure 3B revealing the strong peaks. The XRD peaks of Sample 1 fibers displayed in Figure 3A match closely the cubic-tetragonal structure of $\mathrm{BaTiO}_{3}$ (JCPDS 31-0174 \& JCPDS 05-0626) [2, 19-21]. Sample with $0 \mathrm{wt} \% \mathrm{BaTiO}_{3}$ shows 5 peaks corresponding to PVDF crystal structures at $18.3,20.2,35.5,41.1$ and $56.1^{\circ}[7,22-24]$. The peaks at $18.3^{\circ}$ and $35.6^{\circ}$ (Figure 3A) are attributed to the $\alpha$-crystalline structure of PVDF. The peaks at 20.2, 41.1 and $56.1^{\circ}$ are due to the ferroelectric $\beta$-crystalline phase of PVDF [2, 7]. Evidently, the main peak for neat 

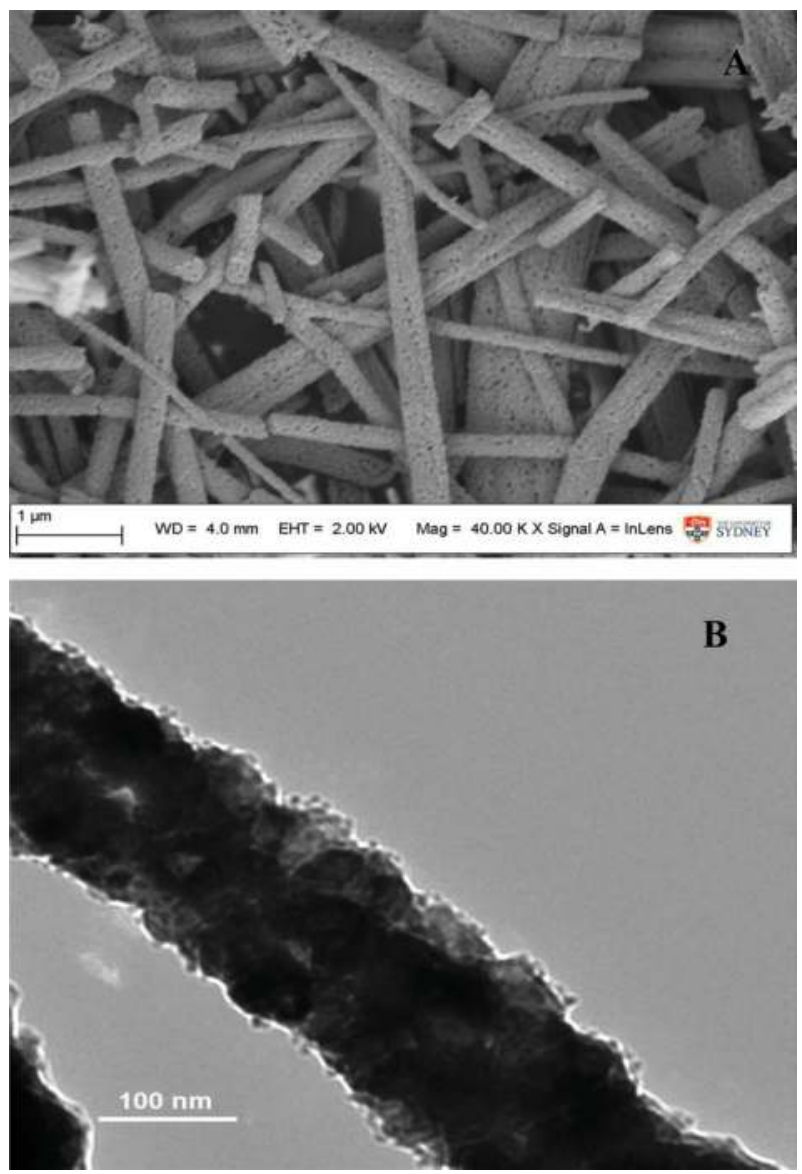

Figure 1. (A) SEM and (B) TEM images of $\mathrm{BaTiO}_{3}$ fiber (Sample 1). The microstructure shows that $\mathrm{BaTiO}_{3}$ grains are selfassembled and self-organized to yield a fibrous geometry.

PVDF is recorded at $20.2^{\circ}$, which is indexed to the $200 / 110$ reflections of the $\beta$-crystalline structure. Nonetheless, XRD peaks corresponding to both $\mathrm{PVDF}$ and $\mathrm{BaTiO}_{3}$ crystalline phases are clearly shown for the fibers reinforced with 10, 20 and $40 \mathrm{wt} \% \mathrm{BaTiO}_{3}$. The intensity of the peak at $20.2^{\circ}$ is moderately increased with $\mathrm{BaTiO}_{3}$ content. Furthermore, the intensities of the peaks related to $\mathrm{BaTiO}_{3}$ are seen to increase and become more prominent with increasing filler content in the composite fibers.

The crystalline structure confirmation for the fibers reinforced with $\mathrm{BaTiO}_{3}$ was done aided by the FTIR spectra recorded for the fibers. Figure 4A shows the IR spectra of Sample 2 fibers. All the samples show peaks at 766, 840, 1280, 1400 and $1432 \mathrm{~cm}^{-1}$. The bands corresponding to 766,1400 and $1432 \mathrm{~cm}^{-1}$ in the spectra are indexed to the $\alpha$-crystals of the PVDF matrix, while the bands at 840 and $1280 \mathrm{~cm}^{-1}$ in the spectra are indexed to the $\beta$-crystals of PVDF $[2,7,23,24]$. These results indicate that the fiber samples irrespective of the $\mathrm{BaTiO}_{3}$ content contain both $\alpha$ and $\beta$ 

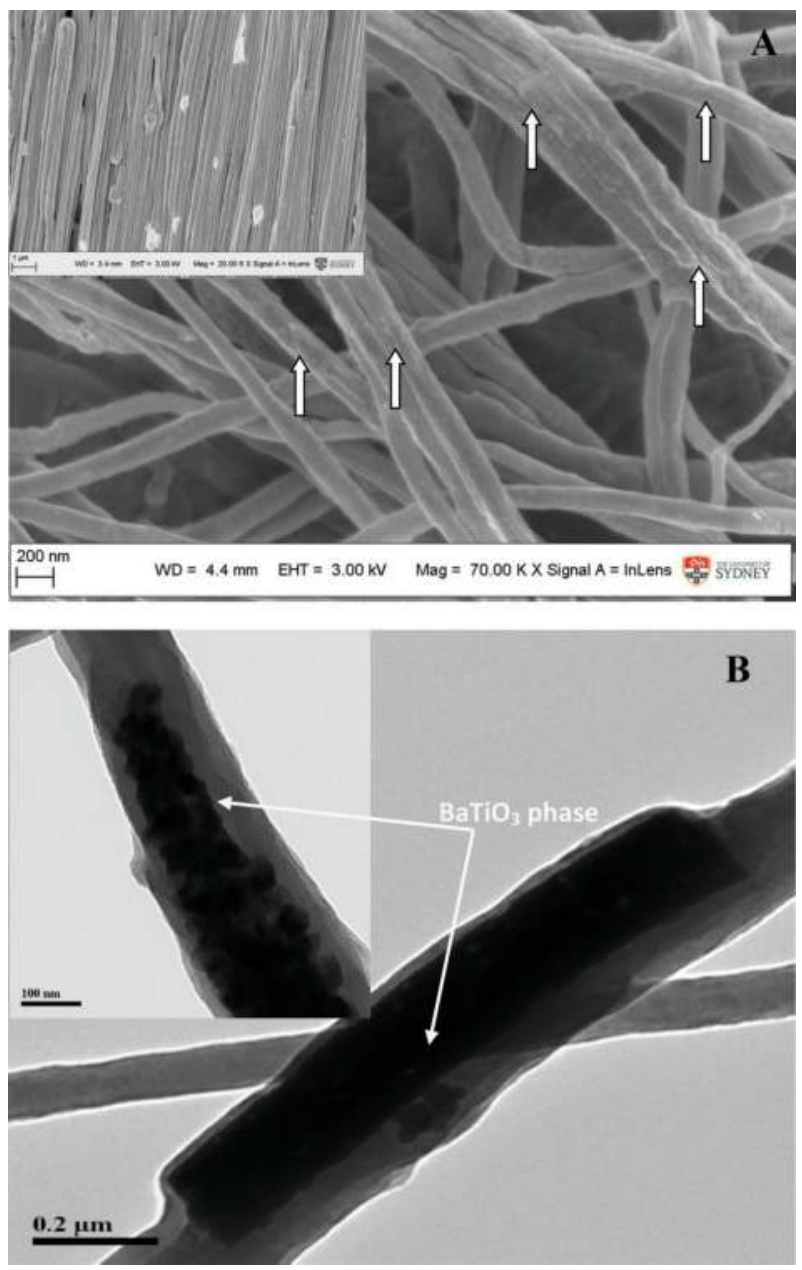

Figure 2. (A) SEM image of PVDF reinforced $\mathrm{BaTiO}_{3}$ fibers (Sample 2). The inset in the figure shows the microstructure of uniaxially aligned fiber arrays. Aligned PVDF fibers as a function of $\mathrm{BaTiO}_{3}$ content were collected for characterizing their tensile properties. (B) TEM images of typical PVDF fibers reinforced with $20 \mathrm{wt} \% \mathrm{BaTiO}_{3}$. It is evident that the $\mathrm{BaTiO}_{3}$ fiber is embedded within the PVDF matrix and aligned along its fiber axis. The inset in the figure shows a higher magnification image taken of another fiber area.

crystals in PVDF matrix. However, the content of $\alpha$ - and $\beta$-crystalline structures inside the PVDF fibers due to the $\mathrm{BaTiO}_{3}$ inclusion is estimated using the Beer-Lambert law. Thus, according to Beer-Lambert law, $\alpha$ - and $\beta$-crystalline content can be estimated by using the absorbencies for $\alpha$ and $\beta$-crystals at 766 and $840 \mathrm{~cm}^{-1}$, respectively. The fraction of $\beta$-phase is calculated from [25, 26]:

$$
\mathrm{F}(\beta)=\frac{A_{\beta}}{\left(1.26 A_{\alpha}+A_{\beta}\right)}
$$



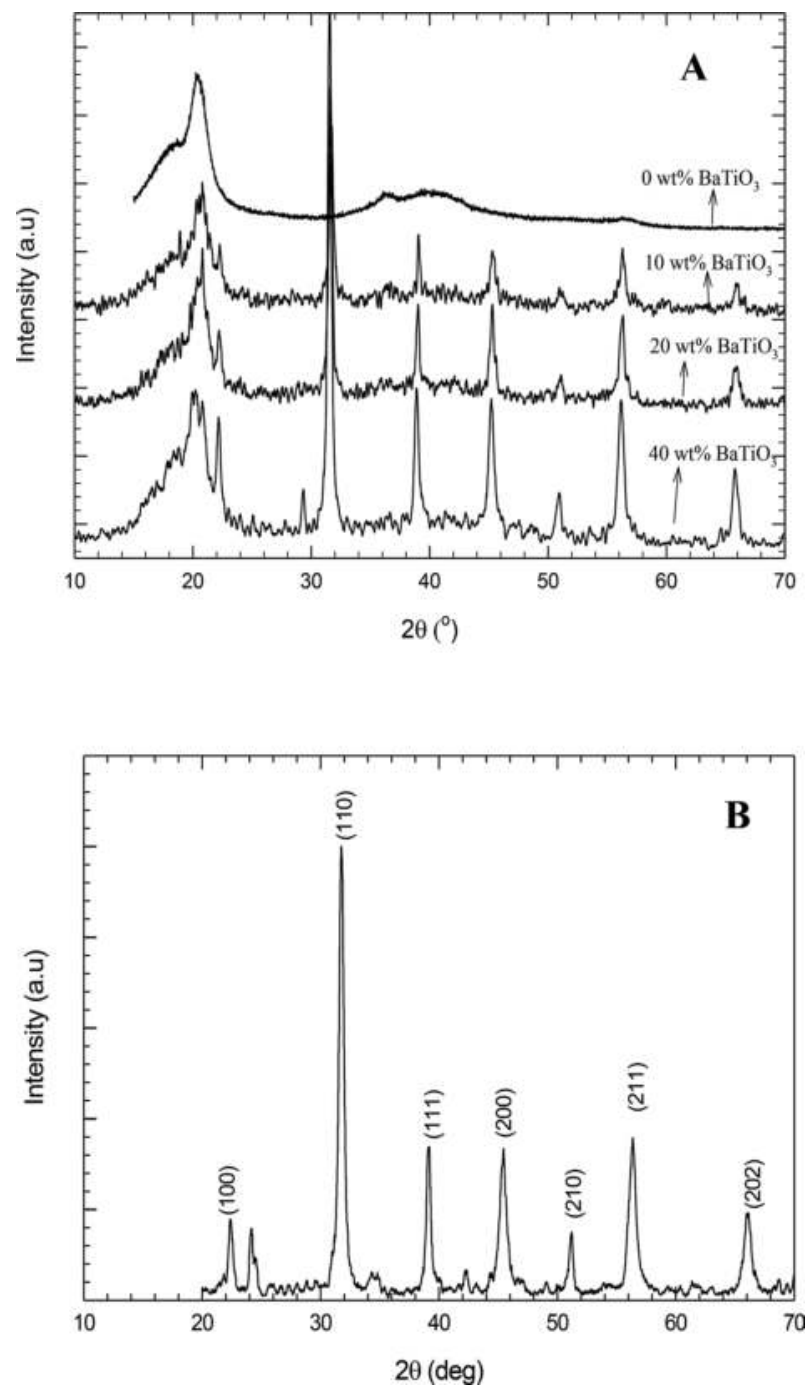

Figure 3. (A) XRD patterns of PVDF fibers as a function of $\mathrm{BaTiO}_{3}$ content. All peaks corresponding to the crystalline phases of $\mathrm{PVDF}$ and $\mathrm{BaTiO}_{3}$ are evident in the fibers reinforced with $\mathrm{BaTiO}_{3}$. (B) XRD pattern of neat $\mathrm{BaTiO}_{3}$ to identify the cubic and tetragonal structure of $\mathrm{BaTiO}_{3}$.

where $A_{\alpha}$ and $A_{\beta}$ are the corresponding absorbency at 766 and $840 \mathrm{~cm}^{-1}$. Figure $4 \mathrm{~B}$ shows the fraction of $\beta$-crystals estimated within the PVDF matrix for Sample 2 fibers. The $\beta$-crystals content within the sample fibers increases from $81 \%$ estimated for neat fibers to $87 \%$ for fibers reinforced with $40 \mathrm{wt} \% \mathrm{BaTiO}_{3}$. This shows that the inclusion of $\mathrm{BaTiO}_{3}$ improves the ferroelectric crystal phase formation within the PVDF fibers. 

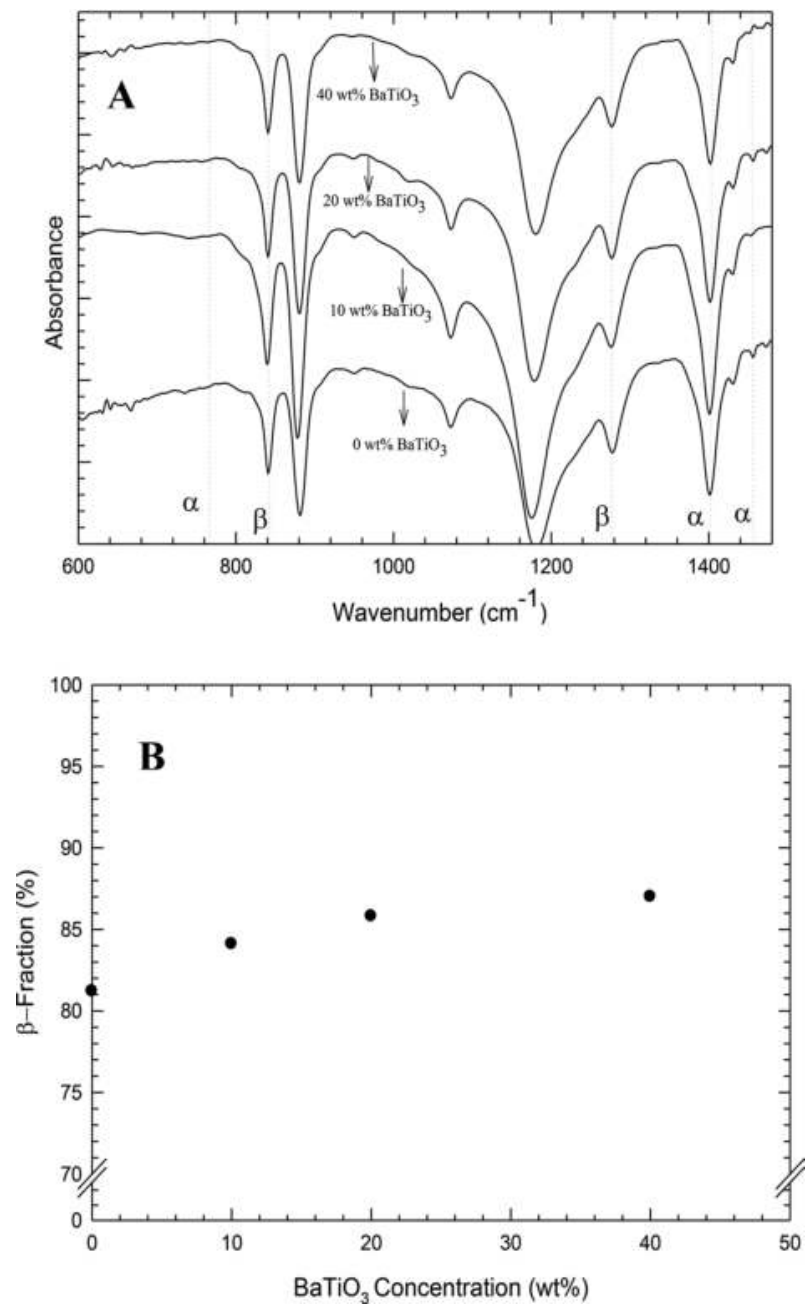

Figure 4. (A) IR spectra of fibers as a function of $\mathrm{BaTiO}_{3}$ content. $\alpha$ - and $\beta$-related bands of PVDF are indexed in the spectra. (B) Fraction of $\beta$-phase as a function of $\mathrm{BaTiO}_{3}$ content. The $\beta$-phase fractions are determined using Eq. (1).

The FTIR data corroborates the XRD results. The improvement in the fraction of $\beta$-crystals within PVDF can be attributed to the changes in crystalline structure development due to the inclusion of $\mathrm{BaTiO}_{3}$. It proves that the $\mathrm{BaTiO}_{3}$ content in PVDF fibers plays an important role in influencing the crystallization of PVDF and also promotes phase change within the PVDF. These results on crystallization induced by $\mathrm{BaTiO}_{3}$ content are in agreement with the results reported by Dang et al. [17]. They show that $\mathrm{BaTiO}_{3}$ can be easily absorbed on surfaces of PVDF due to the presence of interstitial hydrogen ion $\mathrm{BaTiO}_{3}$ lattice. This also helps in the dispersion of $\mathrm{BaTiO}_{3}$ in polar DMF solvent. The homogeneous dispersion and absorption of $\mathrm{BaTiO}_{3}$ on the surface of PVDF plays an important role to induce crystal structure changes [17]. 
We will now characterize the deformation behavior of these PVDF fibers filled with $\mathrm{BaTiO}_{3}$ and also illustrate the mechanisms behind the improved mechanical integrity of the composite fibers. Figure 5A shows typical stress-strain curves for $\mathrm{BaTiO}_{3} / \mathrm{PVDF}$ fibers. The break strain decreases with increasing $\mathrm{BaTiO}_{3}$ content within the fiber. Figure 5B shows the tensile modulus and tensile strength of the composite fibers. In general, as the $\mathrm{BaTiO}_{3}$ content increases, the composite fibers become stiffer and stronger. The stiffness and strength of neat PVDF fibers are increased by 36 and $12 \%$, respectively, when they are filled with $10 \mathrm{wt} \% \mathrm{BaTiO}_{3}$ content. These tensile results of $\mathrm{BaTiO}_{3} / \mathrm{PVDF}$ fibers are contrary to those obtained by Fang et al. [16] on thin films of $\mathrm{BaTiO}_{3} /$ poly(vinyledene fluoride-trifluoroethylene) (P(VDF-TrFE)) [16]. In their study, adding $\mathrm{BaTiO}_{3}$ to $\mathrm{P}(\mathrm{VDF}-\mathrm{TrFE})$ softened and reduced the matrix tensile strength. Reductions in both stiffness
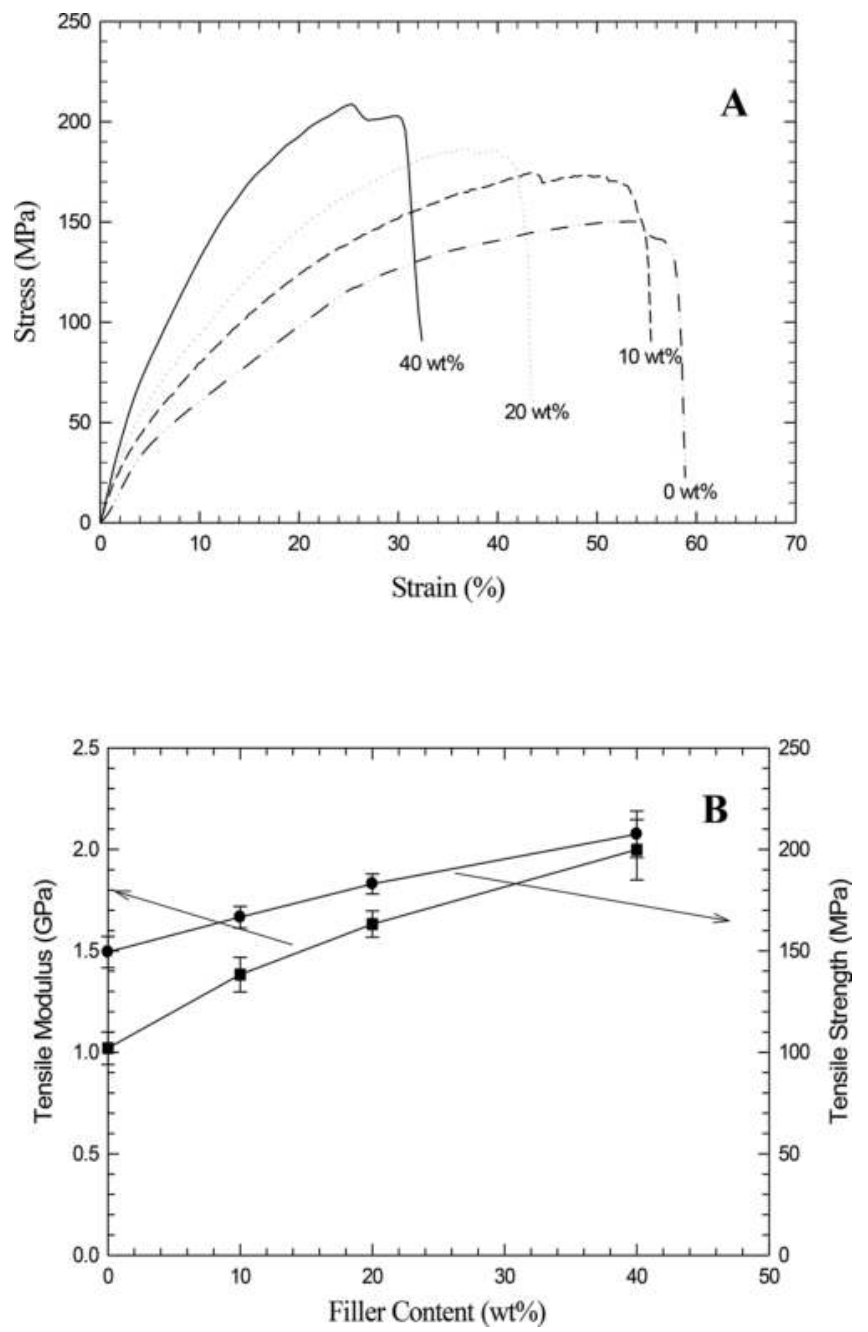

Figure 5. (A) Stress-strain curves obtained from tensile tests conducted on fiber samples as a function of $\mathrm{BaTiO}_{3}$ concentration. (B) Plot of tensile strength and modulus of fiber samples as a function of $\mathrm{BaTiO}_{3}$ content. 
and strength of the composite films are attributed to the weak chemical bonding between the $\mathrm{BaTiO}_{3}$ particles and $\mathrm{P}(\mathrm{VDF}-\mathrm{TrFE})$ matrix and the inhibition of crystallinity due to the addition of $\mathrm{BaTiO}_{3}$ particles. By contrast, we attribute the increase in tensile properties of $\mathrm{BaTiO}_{3} / \mathrm{PVDF}$ fibers to the reinforcement effect of $\mathrm{BaTiO}_{3}$. We employ DMA to investigate the influence of $\mathrm{BaTiO}_{3}$ on the polymeric chain mobility within the fibrous matrix and attribute the increase in strength and stiffness of the fibers to the reinforcement effect of $\mathrm{BaTiO}_{3}$. Figure 6 presents the loss tangent $\left(\tan \delta\right.$ ) as a function of temperature for $\mathrm{BaTiO}_{3} / \mathrm{PVDF}$ fibers. The $\beta$-transition region of the fibers relates to the glass transition temperature $\left(T_{g}\right)$ of the fibers. Comparing the effect of $\mathrm{BaTiO}_{3}$ content on the fibrous matrix, neat PVDF fibers show a peak of the tan $\delta$ versus temperature curve at the lowest temperature $\left(-37.33^{\circ} \mathrm{C}\right)$ while the composite fibers show peaks of the tan $\delta$ versus temperature curves at higher temperatures. For example, fibers filled with 10, 20 and $40 \mathrm{wt} \% \mathrm{BaTiO}_{3}$ show $\mathrm{T}_{g}$ at $-36.06,-35.44$ and $-31.9^{\circ} \mathrm{C}$, respectively. This explains that the mobility of neighboring chains surrounding the $\mathrm{BaTiO}_{3}$ phase is inhibited. In neat PVDF fibers, the chains are relatively free to rotate while in fibers filled with $\mathrm{BaTiO}_{3}$ phase, the $\mathrm{BaTiO}_{3}$ freezes the movement of the chains. The fact that $\mathrm{BaTiO}_{3}$ are adsorbed on PVDF surface may also explain the $\mathrm{BaTiO}_{3}$ inclusion restricts the mobility of PVDF chains.

The effect of $\mathrm{BaTiO}_{3}$ content on the relative permittivity of Sample 2 fibers has been studied over a broad frequency range from the measured capacitance $(C)$ which is given by:

$$
\varepsilon_{r}=\frac{C d}{A \varepsilon_{0}}
$$

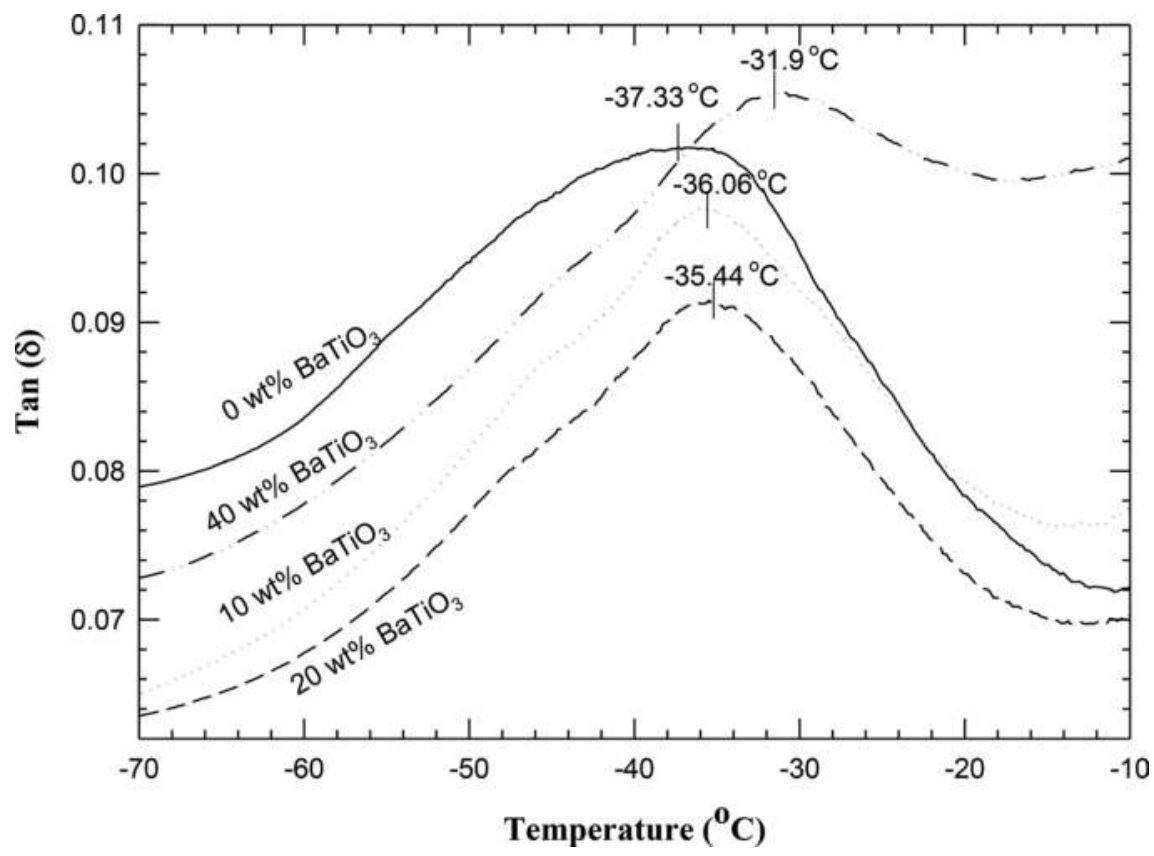

Figure 6. Plot of tan $\delta$ versus temperature obtained using DMA. The effect of $\mathrm{BaTiO}_{3}$ content on the glass transition temperature $\left(T_{g}\right)$ of the fiber is evaluated. 
where $\varepsilon_{r}$ is relative permittivity of capacitor, $d$ thickness of samples, $A$ surface area, and $\varepsilon_{0}$ is free space dielectric constant taken as $8.854 \times 10^{-12} \mathrm{~F} / \mathrm{m}$. The dependence of relative permittivity, $\varepsilon_{r^{\prime}}$ and dielectric loss tangent of $\mathrm{BaTiO}_{3} / \mathrm{PVDF}$ fibers on frequency are shown in Figure 7A and $\mathbf{B}$, respectively. Clearly, the dielectric permittivity increases with $\mathrm{BaTiO}_{3}$ content; but the dielectric
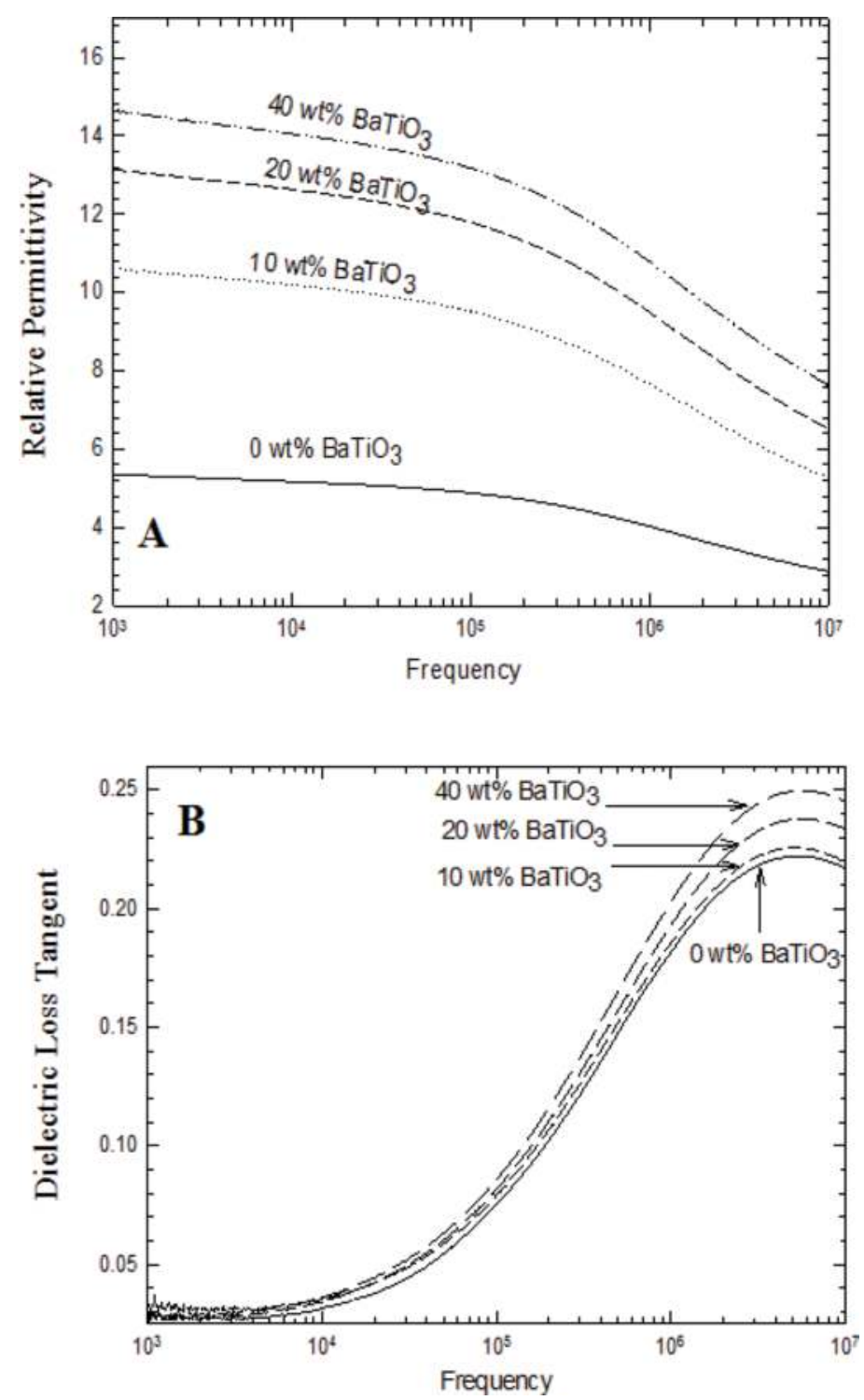

Figure 7. (A) Frequency dependence of effective dielectric constant (measured at $300 \mathrm{~K}$ ) of PVDF fiber as function of $\mathrm{BaTiO}_{3}$ content. (B) Frequency dependence of effective dielectric loss tangent (measured at $300 \mathrm{~K}$ ) of PVDF fiber as functions of $\mathrm{BaTiO}_{3}$ content. 
loss tangent remains nearly the same for all fibers. The relaxation drop in relative permittivity at $\sim 10^{5} \mathrm{~Hz}$ is due to the characteristic dielectric behavior of PVDF matrix, and the rapid drop in dielectric permittivity after $10^{5} \mathrm{~Hz}$ is because the dipole relaxations of the fibers lag behind the fast change of the applied field $[1,4,17]$. Finally, the relative permittivity of fibers with $0,10,20$ and $40 \mathrm{wt} \% \mathrm{BaTiO}_{3}$ at $10^{3} \mathrm{~Hz}$ are 5.4, 10.6, 13.13 and 14.6, respectively (see Figure 7A).

Typically, the dielectric loss of a material should be as low as possible for its use in capacitor applications. Figure 7B compares the dielectric loss tangent of the sample fibers as a function of frequency. Typically, the loss tangent of $\mathrm{BaTiO}_{3}$ reaches a maximum value in the gigahertz and terahertz frequency range and it does not show any significant dielectric losses up to the megahertz frequency range. Thus, the dielectric loss of PVDF fibers filled with $\mathrm{BaTiO}_{3}$ recorded at low frequency (up to $10^{6} \mathrm{~Hz}$ ) is mainly attributed to the loss tangent values of PVDF. A moderate increase in the loss tangent above $10^{6} \mathrm{~Hz}$ frequency is due to the loss tangent contribution arising from the $\mathrm{BaTiO}_{3}$. It is evident from Figure $\mathbf{7 B}$ there is a clear dielectric loss peak at $5 \mathrm{MHz}$ which is attributed to the relaxation loss process of PVDF [17]. These results show that the dielectric properties of $\mathrm{BaTiO}_{3} / \mathrm{PVDF}$ are useful for applications in electronic devices.

\section{Conclusion}

In this study, we use electrospinning to obtain PVDF fibers reinforced with $\mathrm{BaTiO}_{3}$. We demonstrate the effect of $\mathrm{BaTiO}_{3}$ on the crystalline structure developments, tensile and dielectric properties of PVDF fibers. Reinforcing PVDF fibers with $\mathrm{BaTiO}_{3}$ promotes the formation of ferroelectric $\beta$-crystalline phase within the fibers. Tensile strength and stiffness of the fibers increase with $\mathrm{BaTiO}_{3}$ content. Finally, the effective dielectric permittivity and dielectric loss of the fibers increase with $\mathrm{BaTiO}_{3}$ content at all frequencies studied.

\section{Acknowledgements}

We thank the Australian Research Council (ARC) for the continuous support of this project (\#DP0665856).

\section{Author details}

Avinash Baji ${ }^{1 *}$ and Yiu-Wing $\mathrm{Mai}^{2}$

*Address all correspondence to: avinash_baji@sutd.edu.sg

1 Engineering Product Development (EPD) Pillar, Singapore University of Technology and Design (SUTD), Singapore

2 Centre for Advanced Materials Technology (CAMT), School of Aerospace, Mechanical and Mechatronic Engineering, The University of Sydney, Sydney, NSW, Australia 


\section{References}

[1] Bai Y, Cheng ZY, Bharti V, Xu HS, Zhang QM. High-dielectric-constant ceramic-powder polymer composites. Applied Physics Letters. 2000;76:3804-3806

[2] Baji A, Mai Y-W, Li Q, Liu Y. Nanoscale investigation of ferroelectric properties in electrospun barium titanate/polyvinylidene fluoride composite fibers using piezoresponse force microscopy. Composites Science and Technology. 2011;71:1435-1440

[3] Li JJ, Seok SI, Chu BJ, Dogan F, Zhang QM, Wang Q. Nanocomposites of ferroelectric polymers with $\mathrm{TiO}(2)$ nanoparticles exhibiting significantly enhanced electrical energy density. Advanced Materials. 2009;21:217

[4] Damjanovic D. Ferroelectric, dielectric and piezoelectric properties of ferroelectric thin films and ceramics. Reports on Progress in Physics. 1998;61:1267-1324

[5] Takenaka T, Nagata H. Current status and prospects of lead-free piezoelectric ceramics. Journal of the European Ceramic Society. 2005;25:2693-2700

[6] Baji A, Mai Y-W, Li QA, Wong SC, Liu Y, Yao QW. One-dimensional multiferroic bismuth ferrite fibers obtained by electrospinning techniques. Nanotechnology. 2011;22:235702

[7] Buckley J, Cebe P, Cherdack D, Crawford J, Ince BS, Jenkins M, et al. Nanocomposites of poly(vinylidene fluoride) with organically modified silicate. Polymer. 2006;47:2411-2422

[8] Gregorio R, Cestari M. Effect of crystallization temperature on the crystalline phase content and morphology of poly(vinylidene fluoride). Journal of Polymer Science Part B: Polymer Physics. 1994;32:859-870

[9] Kim P, Doss NM, Tillotson JP, Hotchkiss PJ, Pan MJ, Marder SR, et al. High energy density nanocomposites based on surface-modified $\mathrm{BaTiO}(3)$ and a ferroelectric polymer. ACS Nano. 2009;3:2581-2592

[10] Tang HX, Lin YR, Andrews C, Sodano HA. Nanocomposites with increased energy density through high aspect ratio PZT nanowires. Nanotechnology. 2011;22:015702

[11] Andrews C, Lin Y, Sodano HA. The effect of particle aspect ratio on the electroelastic properties of piezoelectric nanocomposites. Smart Materials and Structures. 2010;19:025018

[12] Newnham RE, Skinner DP, Cross LE. Connectivity and piezoelectric-pyroelectric composites. Materials Research Bulletin. 1978;13:525-536

[13] Dias CJ, DasGupta DK. Inorganic ceramic/polymer ferroelectric composite electrets. IEEE Transactions on Dielectrics and Electrical Insulation. 1996;3:706-734

[14] Chen LF, Hong YP, Chen XJ, Wu QL, Huang QJ, Luo XT. Preparation and properties of polymer matrix piezoelectric composites containing aligned $\mathrm{BaTiO}_{3}$ whiskers. Journal of Materials Science. 2004;39:2997-3001

[15] Shen Y, Nan CW, Li M. Anisotropic electrical properties of semiconductive bi(2)S(3) nanorod filled ferroelectric polyvinylidene fluoride. Chemical Physics Letters. 2004;396: $420-423$ 
[16] Fang F, Yang W, Zhang MZ, Wang Z. Mechanical response of barium-titanate/polymer 0-3 ferroelectric nano-composite film under uniaxial tension. Composites Science and Technology. 2009;69:602-605

[17] Dang ZM, Wang HY, Zhang YH, Qi JQ. Morphology and dielectric property of homogenous $\mathrm{BaTiO}_{3} / \mathrm{PVDF}$ nanocomposites prepared via the natural adsorption action of nanosized $\mathrm{BaTiO}_{3}$. Macromolecular Rapid Communications. 2005;26:1185-1189

[18] Yuh J, Nino JC, Sigmund WM. Synthesis of barium titanate $\left(\mathrm{BaTiO}_{3}\right)$ nanofibers via electrospinning. Materials Letters. 2005;59:3645-3647

[19] Kamalasanan MN, Kumar ND, Chandra S. Structural and microstructural evolution of barium titanate thin-films deposited by the sol-gel process. Journal of Applied Physics. 1994;76:4603-4609

[20] Frey MH, Payne DA. Grain-size effect on structure and phase transformations for barium titanate. Physical Review B. 1996;54:3158-3168

[21] Lu SW, Lee BI, Wang ZL, Samuels WD. Hydrothermal synthesis and structural characterization of $\mathrm{BaTiO}_{3}$ nanocrystals. Journal of Crystal Growth. 2000;219:269-276.22

[22] Zheng JF, He AH, Li JX, Han CC. Polymorphism control of poly(vinylidene fluoride) through electrospinning. Macromolecular Rapid Communications. 2007;28:2159-2162

[23] Priya L, Jog JP. Poly(vinylidene fluoride)/clay nanocomposites prepared by melt intercalation: Crystallization and dynamic mechanical behavior studies. Journal of Polymer Science Part B: Polymer Physics. 2002;40:1682-1689

[24] Levi N, Czerw R, Xing SY, Iyer P, Carroll DL. Properties of polyvinylidene difluoridecarbon nanotube blends. Nano Letters. 2004;4:1267-1271

[25] Gregorio R, Cestari M. Effect of crystallization temperature on the crystalline phase content and morphology of poly(vinylidene fluoride). Journal of Polymer Science Part B: Polymer Physics. 1994;32:859-870

[26] Buckley J, Cebe P, Cherdack D, Crawford J, Ince BS, Jenkins M, et al. Nanocomposites of poly(vinylidene fluoride) with organically modified silicate. Polymer. 2006;47:2411-2422 